\title{
Iterated function systems with a weak separation condition
}

\author{
by \\ KA-Sing LAU (Hong Kong) and \\ Xiang-Yang Wang (Hong Kong and Guang-Zhou)
}

\begin{abstract}
Nonoverlapping contractive self-similar iterated function systems (IFS) have been studied in great detail via the open set condition. On the other hand much less is known about IFS with overlaps. To deal with such systems, a weak separation condition (WSC) has been introduced recently [LN1]; it is weaker than the open set condition and it includes many important overlapping cases. This paper has two purposes. First, we consider the class of self-similar measures generated by such IFS; we give a necessary and sufficient condition for the self-similar measures to be absolutely continuous with respect to Hausdorff measures. This extends a result in [LNR]. As most of the known examples of the WSC involve algebraic integers (e.g., the golden ratio, integral dilation matrices) and the contraction ratios are equal, our second goal is to give new examples of self-similar IFS with the WSC and with more arbitrary contraction ratios.
\end{abstract}

1. Introduction. We call a family $\left\{S_{j}\right\}_{j=1}^{N}$ of contractive maps on $\mathbb{R}^{d}$ an iterated function system (IFS). It is well known that there exists a unique nonempty compact subset $K \subset \mathbb{R}^{d}$ such that $K=\bigcup_{j=1}^{N} S_{j}(K)$. We call $K$ the invariant set or attractor of the IFS. Furthermore, if we associate with the IFS a set $\left\{p_{j}\right\}_{j=1}^{N}$ of probability weights, then there exists a unique probability measure $\mu$ supported by $K$ and satisfying

$$
\mu(A)=\sum_{j=1}^{N} p_{j} \mu \circ S_{j}^{-1}(A)
$$

for all Borel sets $A \subset \mathbb{R}^{d}$ [Hut]. We call $\mu$ the invariant measure of the IFS with respect to the weights $\left\{p_{j}\right\}_{j=1}^{N}$. As is well known the invariant measure is either continuously singular or absolutely continuous with respect to the Lebesgue measure on $\mathbb{R}^{d}$.

In order to obtain sharp results on the invariant measures, it is often assumed that the maps $\left\{S_{j}\right\}_{j=1}^{N}$ are similitudes. The corresponding $K$ and $\mu$

2000 Mathematics Subject Classification: Primary 28A80; Secondary 42B10.

Key words and phrases: absolute continuity, self-similar measure, iterated function system, weak separation condition.

Research supported in part by the HKRGC Grant. 
in (1.1) are called the self-similar set and self-similar measure respectively. For the iteration, it is often assumed that the IFS satisfies the open set condition (OSC), i.e., there exists a nonempty bounded open set $O \subset \mathbb{R}^{d}$ such that

$$
\bigcup_{j=1}^{N} S_{j}(O) \subset O, \quad S_{i}(O) \cap S_{j}(O)=\emptyset \quad \forall i \neq j .
$$

In this case the $S_{j}(K)$ 's are "essentially" disjoint. The advantage of this is that the IFS on the set $K$ can be identified with the shift map (actually the branches of the inverse of the shift map) on a symbolic space, and many properties can be derived from there. Without the OSC, the $S_{j}(K)$ 's have overlaps. The identification thus fails and it is more difficult to handle the situation. For example, even the simplest case

$$
S_{1}(x)=r x, \quad S_{2}(x)=r x+(1-r),
$$

with $1 / 2<r<1$ and $\mu_{r}=\frac{1}{2} \mu_{r} \circ S_{1}^{-1}+\frac{1}{2} \mu_{r} \circ S_{2}^{-1}$, is not completely understood. The measure $\mu_{r}$ is referred to as the Bernoulli convolution because of its origin from the consideration of the discount sum $\sum_{n=1}^{\infty} r^{n} X_{n}$, where the $X_{n}$ 's are i.i.d. Bernoulli random variables [E], [G]. This is the most basic example for overlapping IFS; the details of this can be found in the excellent survey article by Peres, Solomyak and Schlag [PSS] and the references therein.

One direction to study the overlapping IFS is to use a density argument on the parameters of the families of IFS ([PoSi], [S], [PS1,2], [PSiS]). In another direction Lau and Ngai [LN1] introduced a weak separation condition (WSC) which is weaker than the open set condition and includes many IFS with overlaps; in particular the above case of Bernoulli convolution with $r=(\sqrt{5}-1) / 2$ satisfies that condition. Under that condition the absolute continuity of the self-similar measures was studied in [LNR], the general mulitfractal structure was considered in [LN1] and many special cases have been analyzed in detail in [LN2, 3], [FLN], [HL], [LW]. Note also that Zerner [Z] gave a few equivalent definitions of the WSC. We will introduce another equivalent definition which is most convenient for our purpose and it seems to be adaptable to the nonlinear case.

Throughout the paper we assume that $\left\{S_{j}\right\}_{j=1}^{N}$ is an IFS of contractive similitudes on $\mathbb{R}^{d}$ with contraction ratios $0<r_{j}<1$. Let $\Sigma^{*}=$ $\bigcup_{n=0}^{\infty}\{1, \ldots, N\}^{n}$ be the space of finite sequences (by convention $\{1, \ldots, N\}^{0}$ $=\emptyset, S_{\emptyset}$ is the identity map, $r_{\emptyset}=1$ and $\left.p_{\emptyset}=1\right)$. For $J=j_{1} \ldots j_{n} \in \Sigma^{*}$, let $|J|=n$ denote the length of $J, S_{J}=S_{j_{1}} \circ \ldots \circ S_{j_{n}}, r_{J}=r_{j_{1}} \ldots r_{j_{n}}$ and $p_{J}=p_{j_{1}} \ldots p_{j_{n}}$. For $0<b<1$, we let

$$
\mathcal{J}_{b}=\left\{J=j_{1} \ldots j_{n} \in \Sigma^{*}: r_{J} \leq b<r_{j_{1} \ldots j_{n-1}}\right\} .
$$


Note that $S_{J}$ may be equal to $S_{J^{\prime}}$ for some $J \neq J^{\prime}$. We let

$$
\mathcal{A}_{b}=\left\{S: S=S_{J}, J \in \mathcal{J}_{b}\right\} .
$$

In this way we can consider the distinct $S$ 's in $\mathcal{A}_{b}$ and each $S$ has contraction ratio around $b$. For $S \in \mathcal{A}_{b}$, we denote by $r_{S}$ the contraction ratio of $S$. Note that $b r_{\min }<r_{S} \leq b$, where $r_{\min }=\min \left\{r_{j}: j=1, \ldots, N\right\}$. Let $p_{S}=\sum\left\{p_{J}: S_{J}=S, J \in \mathcal{J}_{b}\right\}$. It is well known that the self-similar identity (1.1) can be written as $\mu=\sum_{S \in \mathcal{A}_{b}} p_{S} \mu \circ S^{-1}$.

We say that $\left\{S_{j}\right\}_{j=1}^{N}$ satisfies the weak separation condition (WSC) if there exist $\gamma>0$ and a compact subset $D \subset \mathbb{R}^{d}$, with $D^{\circ} \neq \emptyset$ and $\bigcup_{j=1}^{N} S_{j}(D) \subset D$, such that for any $0<b<1$ and $x \in \mathbb{R}^{d}$,

$$
\#\left\{S \in \mathcal{A}_{b}: x \in S(D)\right\} \leq \gamma .
$$

This definition yields a kind of finite covering at any point $x$ by the $S(D)$, where $S \in \mathcal{A}_{b}$ and $S(D)$ has diameter of the same order as $b$. It generalizes the open set condition; it has also been used in [LNR] extensively as a major property derived from the original definition of the WSC. In the following we will see that they actually coincide through the equivalent conditions of Zerner [Z]. Our main theorem is

THEOREM 1.1. Let $\left\{S_{j}\right\}_{j=1}^{N}$ be contractive similitudes satisfying the WSC. Let $K$ be the self-similar set and let $\mu$ be the self-similar measure defined by (1.1). Then $\mu$ is singular with respect to $\left.\mathcal{H}^{\alpha}\right|_{K}\left(\alpha=\operatorname{dim}_{\mathrm{H}} K\right)$ if and only if there exist $b>0$ and $S \in \mathcal{A}_{b}$ such that $r_{S}^{\alpha}<p_{S}$.

The theorem gives a very convenient criterion to check the singularity of a self-similar measure under the WSC (see $\S 4$ ). In particular for $\alpha=d$, the theorem gives a necessary and sufficient condition for the absolute continuity with respect to the Lebesgue measure. For the WSC, there is no formula for $\alpha=\operatorname{dim}_{\mathrm{H}} K$ analogous to the one in the case of the open set condition $[\mathrm{F}]$. However, for certain classes (e.g., when the $S_{j}^{-1}$ 's are integral self-similar maps), there are algorithms to calculate such $\alpha$ ([HeLR], [NWa]). In the proof of the theorem, the more difficult part is the sufficiency; it has been considered in $[\mathrm{LNR}]$ for the special case where $r_{1}=\ldots=r_{N}$ and for the Lebesgue measure. Here we will adopt a similar method with some modifications.

As an important consequence of the theorem we have

THEOREM 1.2. Under the above assumptions, if the self-similar measure $\mu$ is absolutely continuous with respect to $\left.\mathcal{H}^{\alpha}\right|_{K}$, then the Radon-Nikodym derivative of $\mu$ is bounded.

We remark that if we do not assume the WSC in Theorem 1.1, then it is possible that the self-similar measure $\mu$ is absolutely continuous and the condition $r_{S}^{\alpha}<p_{S}$ is satisfied; there are examples among the Bernoulli 
convolutions [PS1]. However the corresponding Radon-Nikodym derivatives are unbounded [HLW].

To the best of our knowledge, in most existing examples of WSC, the maps are constructed using the integers or algebraic integers [LNR]; also in all these cases, they have equal contraction ratios, or exponentially commensurable at the best, i.e., there exists a constant $0<\varrho<1$ such that $r_{j}=\varrho^{k_{j}}$ for some $k_{j} \in \mathbb{N}, j=1, \ldots, N$. It has been suggested that IFS's satisfying the WSC might just be like that. One of our main purposes in this paper is to disprove this by constructing new examples of the WSC with more general contraction ratios. For such IFS we also provide a calculation of the dimension of the self-similar sets.

The organization of the paper is as follows. We begin in $\S 2$ with some explanations of the new definition. We prove the two theorems in $\S 3$. In $\S 4$, we give some illustrations of the theorems and study in detail a new class of IFS with the WSC. The last section is devoted to some remarks; in particular we discuss the possible extension of the WSC to nonlinear maps.

2. Preliminaries. Throughout the paper we assume that the invariant set $K$ is in general position, i.e., $K$ is not contained in a hyperplane, which is needed in the proof of the equivalence of (ii), (iii) and (iv) in Proposition 2.1. We will use $\mathcal{L}$ to denote the Lebesgue measure on $\mathbb{R}^{d}, E^{\circ}$ the interior of $E$, and $|E|$ the diameter of $E$. Our first goal is to clarify the definition of WSC in (1.2) in comparison with the previous considerations in [LN1], $[\mathrm{LNR}]$ and $[\mathrm{Z}]$. For any $a>0$ and any bounded subsets $U$ and $D$ in $\mathbb{R}^{d}$, we let

$$
\mathcal{A}_{a, U, D}=\left\{S \in \mathcal{A}_{a|U|}: S(D) \cap U \neq \emptyset\right\}, \quad \gamma_{a, D}=\sup _{U} \# \mathcal{A}_{a, U, D} .
$$

Proposition 2.1. Let $\left\{S_{j}\right\}_{j=1}^{N}$ be contractive similutudes. Then the following are equivalent:

(i) there exist $\gamma \in \mathbb{N}$ and a compact subset $D \subset \mathbb{R}^{d}$ with $D^{\circ} \neq \emptyset$ such that $\#\left\{S \in \mathcal{A}_{b}: x \in S(D)\right\} \leq \gamma$ for any $0<b<1$ and $x \in \mathbb{R}^{d}$;

(ii) there exist $x_{0} \in \mathbb{R}^{d}$ and $\gamma \in \mathbb{N}$ such that for any $J \in \Sigma^{*}$, any ball of radius $b$ contains at most $\gamma$ points of $\left\{S\left(S_{J}\left(x_{0}\right)\right): S \in \mathcal{A}_{b}\right\}$;

(iii) there exist $a>0$ and a nonempty bounded subset $D \subset \mathbb{R}^{d}$ such that $\gamma_{a, D}<\infty$

(iv) for any $a>0$ and for any nonempty bounded subset $D \subset \mathbb{R}^{d}$, $\gamma_{a, D}<\infty$.

Proof. Note that (ii) is a simple variation of the original definition of the WSC in [LN1]; the equivalence of (ii), (iii), (iv) was proved in [Z].

(i) $\Rightarrow$ (iii). We first strengthen statement (i) slightly: there exists $\gamma^{\prime}$ such that for any $x \in \mathbb{R}^{d}$, 


$$
\#\left\{S \in \mathcal{A}_{b}: S(D) \cap B_{b}(x) \neq \emptyset\right\} \leq \gamma^{\prime},
$$

where $B_{b}(x)$ is the open ball with radius $b$ and centered at $x$. Indeed, if we let $\eta=(1+|D|) b$, then $S(D) \subset B_{\eta}(x)$. By (i), each point in $D$ is covered by at most $\gamma$ of the $S(D), S \in \mathcal{A}_{b}$. It follows that

$$
\sum\left\{\mathcal{L}(S(D)): S \in \mathcal{A}_{b}, S(D) \cap B_{b}(x) \neq \emptyset\right\} \leq \gamma \mathcal{L}\left(B_{\eta}(x)\right) .
$$

Note that $\left(r_{\min } b\right)^{d} \mathcal{L}(D) \leq r_{S}^{d} \mathcal{L}(D)=\mathcal{L}(S(D))$ and $\mathcal{L}\left(B_{\eta}(x)\right)=c \eta^{d}$ for some constant $c>0$. Since $D^{\circ} \neq \emptyset$, we have $\mathcal{L}(D)>0$ and by letting $\gamma^{\prime}=\gamma c(1+|D|)^{d}\left(r_{\text {min }}^{d} \mathcal{L}(D)\right)^{-1}$, the above inequality implies the claim.

Now to prove (iii), we choose $a=1$ and let $D$ be as given in (i). Let $U$ be any bounded set, and let $B$ be a ball of radius $|U|$ and containing $U$. Then

$$
\left\{S \in \mathcal{A}_{|U|}: S(D) \cap U \neq \emptyset\right\} \subset\left\{S \in \mathcal{A}_{|U|}: S(D) \cap B \neq \emptyset\right\} .
$$

By applying the claim for $b=|U|$, we have $\gamma_{1, D}=\sup _{U} \# \mathcal{A}_{1, U, D} \leq \gamma^{\prime}$.

(iv) $\Rightarrow$ (i). Let $D$ be any fixed compact subset in $\mathbb{R}^{d}$. Then for any $x \in \mathbb{R}^{d}$ and $0<b<1$,

$$
\begin{aligned}
\#\left\{S \in \mathcal{A}_{b}: x \in S(D)\right\} & \leq \#\left\{S \in \mathcal{A}_{b}: S(D) \cap B_{b / 2}(x) \neq \emptyset\right\} \\
& \leq \# \mathcal{A}_{1, B_{b / 2}(x), D} \leq \gamma_{1, D}<\infty .
\end{aligned}
$$

The last proof actually yields

Corollary 2.2. Condition (i) is equivalent to: For any compact set $D$, there exists $\gamma$ (depending on $D$ ) such that $\#\left\{S \in \mathcal{A}_{b}: x \in S(D)\right\} \leq \gamma$ for any $0<b<1$ and $x \in \mathbb{R}^{d}$.

In view of this we see that the choice of $D$ in the definition of weak separation condition in (1.1) is quite flexible. We assume $D$ to be compact, $D^{\circ} \neq \emptyset$ and $\bigcup_{j=1}^{N} S_{j}(D) \subset D$ because this implies that the invariant set $K$ is contained in $D$.

An important consequence of the WSC is

Proposition 2.3. Suppose $\left\{S_{j}\right\}_{j=1}^{N}$ satisfies the WSC and let $\alpha=$ $\operatorname{dim}_{\mathrm{H}} K$. Then $0<\mathcal{H}^{\alpha}(K)<\infty$ and there exists a constant $C_{1}>0$ such that for any $0<b<1$,

$$
\# \mathcal{A}_{b} \leq C_{1} b^{-\alpha} \text {. }
$$

Proof. That $0<\mathcal{H}^{\alpha}(K)<\infty$ is proved in [Z]. For (2.1), we adopt a similar proof as in the last proposition. Note that for any $0<b<1$, $K=\bigcup\left\{S(K): S \in \mathcal{A}_{b}\right\}$. By the definition of the WSC, we know that $K \subset D$ and each $x \in K$ is covered by at most $\gamma$ of the $S(K)$ with $S \in \mathcal{A}_{b}$. Hence

$$
\sum_{S \in \mathcal{A}_{b}} \mathcal{H}^{\alpha}(S(K)) \leq \gamma \mathcal{H}^{\alpha}(K)
$$


For each $S \in \mathcal{A}_{b}$, it is clear that $r_{\min } b \leq r_{S}$; hence $\left(r_{\min } b\right)^{\alpha} \mathcal{H}^{\alpha}(K) \leq$ $r_{S}^{\alpha} \mathcal{H}^{\alpha}(K)=\mathcal{H}^{\alpha}(S(K))$. It follows that

$$
\left(r_{\min } b\right)^{\alpha} \mathcal{H}^{\alpha}(K) \# \mathcal{A}_{b} \leq \gamma \mathcal{H}^{\alpha}(K)
$$

and by making use of $0<\mathcal{H}^{\alpha}(K)<\infty$, the assertion follows.

Proposition 2.4. Suppose $\left\{S_{j}\right\}_{j=1}^{N}$ satisfies the WSC. Then for any finite subset $\Lambda \subset \Sigma^{*}$, the family $\left\{S_{J}: J \in \Lambda\right\}$ also satisfies the WSC.

Proof. We will first prove the proposition for the family $\left\{S_{12}, S_{1}, S_{2}, \ldots\right.$ $\left.\ldots, S_{N}\right\}$. For convenience, we write $S_{0}=S_{12}$. Let $\mathcal{J}_{b}^{\prime}$ and $\mathcal{A}_{b}^{\prime}$ be defined with respect to the index set $\{0,1, \ldots, N\}$.

Let $J=j_{1} \ldots j_{k} \in \mathcal{J}_{b}^{\prime}$. Then $r_{j_{1} \ldots j_{k}} \leq b<r_{j_{1} \ldots j_{k-1}}$. If $j_{k} \neq 0$, then it is trivial that $J \in \mathcal{J}_{b}$; if $j_{k}=0$, then by noting that $r_{0}=r_{1} r_{2}$, the inequality

$$
r_{j_{1} \ldots j_{k-1}} r_{1} r_{2} \leq b<r_{j_{1} \ldots j_{k-1}}
$$

implies that either

$$
r_{j_{1} \ldots j_{k-1}} r_{1} r_{2} \leq b<r_{j_{1} \ldots j_{k-1}} r_{1} \quad \text { or } \quad r_{j_{1} \ldots j_{k-1}} r_{1} \leq b<r_{j_{1} \ldots j_{k-1}},
$$

i.e., either $S \in \mathcal{A}_{b}$ or $S=\widetilde{S} \circ S_{2}$ with $\widetilde{S} \in \mathcal{A}_{b}$. Thus

$$
\mathcal{A}_{b}^{\prime} \subset \mathcal{A}_{b} \cup \mathcal{A}_{b} \circ S_{2} \text {. }
$$

It follows that for any $x \in D$,

$$
\begin{aligned}
\#\left\{S \in \mathcal{A}_{b}^{\prime}: x \in S(D)\right\} \leq & \#\left\{S \in \mathcal{A}_{b}: x \in S(D)\right\} \\
& +\#\left\{S \in \mathcal{A}_{b}: x \in S \circ S_{2}(D)\right\} \leq 2 \gamma
\end{aligned}
$$

(note that $S_{2}(D) \subset D$ ). Hence the proposition is true for $\Lambda=\{12,1,2, \ldots$ $\ldots, N\}$. By repeating this argument, we see that it is also true for $\Lambda=$ $\bigcup_{k=1}^{n}\{1, \ldots, N\}^{k}$. The general statement follows by observing the trivial fact that if an IFS satisfies the WSC, then so does any subfamily of the IFS.

3. The theorems. We need to introduce more notations. Let $\Sigma^{*}$ be the space of finite indices as defined earlier and let $\Sigma=\{1, \ldots, N\}^{\mathbb{N}}$. For $J \in \Sigma^{*}$, let $C_{J}$ be the cylinder set in $\Sigma$ and for $\Lambda \subset \Sigma^{*}$, let $C_{\Lambda}=\bigcup\left\{C_{J}: J \in \Lambda\right\}$; for $J \in \Sigma^{*}$, let $[J]=\left\{J^{\prime} \in \Sigma^{*}: S_{J}=S_{J^{\prime}}\right\}$. Let $P$ be the product probability measure on $\Sigma$ induced by the weights $\left\{p_{j}\right\}_{j=1}^{N}$. For $\Lambda \subset \mathcal{J}_{b}$, we will use the abbreviated notation $P(\Lambda)$ to denote $P\left(C_{\Lambda}\right)$. Let $\pi$ be the projection of $\Sigma$ to $\mathbb{R}^{d}$ defined by

$$
\pi(J)=\bigcap_{n=1}^{\infty} S_{j_{1} \ldots j_{n}}(K), \quad J=j_{1} j_{2} \ldots
$$

Then $\pi\left(C_{J}\right)=S_{J}(K)$. Note that $\mu=P \circ \pi^{-1}$. Hence if $S=S_{J}$, then

$$
\mu(S(K))=P\left(\pi^{-1} S(K)\right) \geq P\left(\left\{C_{J^{\prime}}: S_{J^{\prime}}=S\right\}\right)=\sum_{J^{\prime} \in[J]} p_{J^{\prime}}
$$


Lemma 3.1. For $\Lambda \subset \mathcal{J}_{b}$, let

$$
\widetilde{\Lambda}=\left\{J \in \Lambda: \sum_{J^{\prime} \in[J] \cap \Lambda} p_{J^{\prime}}>\frac{b^{\alpha}}{4 C_{1}}\right\},
$$

where $C_{1}$ is as in Proposition 2.3. Then $P(\Lambda)>1 / 2$ implies that $P(\widetilde{\Lambda})>$ $1 / 4$.

Proof. By Proposition 2.3, we have $\# \mathcal{A}_{b} \leq C_{1} b^{-\alpha}$. It follows that

$$
\begin{aligned}
P(\Lambda \backslash \widetilde{\Lambda}) & =\sum\left\{p_{J}: J \in \Lambda \backslash \widetilde{\Lambda}\right\}=\sum_{[J]} \sum_{J^{\prime} \in[J]}\left\{p_{J^{\prime}}: J^{\prime} \in \Lambda \backslash \widetilde{\Lambda}\right\} \\
& \leq \# \mathcal{A}_{b} \cdot \frac{b^{\alpha}}{4 C_{1}} \leq \frac{1}{4}
\end{aligned}
$$

and $P(\widetilde{\Lambda})=P(\Lambda)-P(\Lambda \backslash \widetilde{\Lambda})>1 / 2-1 / 4=1 / 4$.

Proof of Theorem 1.1. To prove the necessity, we suppose that $\mu$ is singular with respect to $\left.\mathcal{H}^{\alpha}\right|_{K}$. Then there exists $K_{0} \subset K$ such that $\mu\left(K_{0}\right)=1$ but $\mathcal{H}^{\alpha}\left(K_{0}\right)=0$. Thus for any $\varepsilon>0$, there exists a $\delta$-cover $\left\{U_{i}\right\}$ of $K_{0}$ such that $\sum_{i}\left|U_{i}\right|^{\alpha}<\varepsilon$. Let $b_{i}=\left|U_{i}\right|$; then $U_{i} \subset B_{b_{i}}\left(x_{i}\right):=B_{i}$, where $x_{i}$ is any fixed element in $U_{i}$. Note that

$$
\begin{aligned}
\mu\left(B_{i}\right) & =\sum\left\{p_{S} \mu \circ S^{-1}\left(B_{i}\right): S \in \mathcal{A}_{b_{i}}, S(K) \cap B_{i} \neq \emptyset\right\} \\
& \leq \sum\left\{p_{S}: S \in \mathcal{A}_{b_{i}}, S(K) \cap B_{i} \neq \emptyset\right\} .
\end{aligned}
$$

If the necessity is not true, then $p_{S} \leq r_{S}^{\alpha} \leq b^{\alpha}$ for all $S \in \mathcal{A}_{b}$ and all $b>0$. Hence the above inequality and Proposition 2.1(iv) imply that $\mu\left(B_{i}\right) \leq C b_{i}^{\alpha}$ and

$$
1=\mu\left(K_{0}\right) \leq \sum_{i} \mu\left(B_{i}\right) \leq C \sum_{i} b_{i}^{\alpha}<C \varepsilon .
$$

Since $C$ is independent of $\varepsilon$, the right side can be arbitrarily small, which is a contradiction.

For the sufficiency, the proof follows the same technique of [LNR], where all the contraction ratios are equal and the singularity is with respect to the Lebesgue measure. For completeness we include a modified proof here. Our aim is to choose, for any $\varepsilon>0$, a subset $E \subset K$ such that $\mu(E) \geq 1 / 2$ and $\mathcal{H}^{\alpha}(E)<\varepsilon$. Without loss of generality we assume that $b=1$ and $p_{1}>r_{1}^{\alpha}$. Otherwise, we can replace $\left\{S_{j}\right\}_{j=1}^{N}$ with $\mathcal{A}_{b}$ and rearrange the indices such that $S$ is the first map in $\mathcal{A}_{b}$ and satisfies $p_{S}>r_{S}^{\alpha}$.

Let $q \in \mathbb{N}$ be such that

$$
4 C_{1} \mathcal{H}^{\alpha}(K)\left(\frac{r_{1}^{\alpha}}{p_{1}}\right)^{q}<\varepsilon
$$


where $C_{1}$ is as in Lemma 3.1. Let $\Lambda_{1}=\{1, \ldots, N\}$ and $\widetilde{\Lambda}_{1}=\left\{j \in \Lambda_{1}: p_{j}>\right.$ $\left.1 /\left(4 C_{1}\right)\right\}$. Lemma 3.1 implies that $P\left(\tilde{\Lambda}_{1}\right) \geq 1 / 4$.

Let $\eta=r_{1}^{q}$. We extend the index $j \in \widetilde{\Lambda}_{1}$ to $j 1 \ldots 1 \in \mathcal{J}_{\eta}$, and denote this set by $\Lambda_{1}^{*}$, i.e.,

$$
\Lambda_{1}^{*}=\left\{j 1^{n} \in \mathcal{J}_{\eta}: j \in \widetilde{\Lambda}_{1}\right\}
$$

(the construction of $\Lambda_{1}^{*}$ and $\Lambda_{k}^{*}$ in the following makes use of the sufficient condition $r_{1}^{\alpha}<p_{1}$ and is the key step in the proof). Note that for $j 1^{n} \in \mathcal{J}_{\eta}$, we have $r_{j} r_{1}^{n} \leq \eta=r_{1}^{q}<r_{j} r_{1}^{n-1}$. It follows that $n \leq q$. Also

$$
P\left(\Lambda_{1}^{*}\right) \geq P\left(\Lambda_{1}\right) p_{1}^{q} \geq \frac{1}{4} p_{1}^{q}
$$

and for $J \in \Lambda_{1}^{*}, p_{J}>\frac{1}{4 C_{1}} p_{1}^{q}>\left(r_{1}^{\alpha q} / \varepsilon\right) \mathcal{H}^{\alpha}(K)$. If we let $E_{1}=\bigcup\left\{S_{J}(K)\right.$ : $\left.J \in \Lambda_{1}^{*}\right\}$, then

$$
\mathcal{H}^{\alpha}\left(E_{1}\right) \leq r_{1}^{\alpha q} \mathcal{H}^{\alpha}(K) \cdot \#\left\{S_{J}: J \in \Lambda_{1}^{*}\right\} \leq \varepsilon \sum_{J \in \Lambda_{1}^{*}} p_{J} \leq \varepsilon P\left(\Lambda_{1}^{*}\right)
$$

Suppose that for $1 \leq i \leq k-1$ we have chosen $\Lambda_{i}^{*} \subset \mathcal{J}_{\eta^{i}}$, and $E_{i}=$ $\bigcup\left\{S_{J}(K): J \in \Lambda_{i}^{*}\right\}$ such that

(i) $C_{\Lambda_{i}^{*}} \cap C_{\Lambda_{j}^{*}}=\emptyset$ for $j<i$;

(ii) $P\left(\Lambda_{i}^{*}\right) \geq \frac{1}{4} p_{1}^{q}$;

(iii) $\mathcal{H}^{\alpha}\left(E_{i}\right) \leq \varepsilon P\left(\Lambda_{i}^{*}\right)$.

If $\sum_{i=1}^{k-1} P\left(\Lambda_{i}^{*}\right) \geq 1 / 2$, we stop the construction. Otherwise $\sum_{i=1}^{k-1} P\left(\Lambda_{i}^{*}\right)<$ $1 / 2$; we let $\left.J\right|_{n}$ denote the first $n$ coordinates of $J$ and define

$$
\begin{aligned}
& \Lambda_{k}=\left\{J \in \mathcal{J}_{\eta^{k-1}}:\left.J\right|_{i} \notin \bigcup_{j=1}^{k-1} \Lambda_{j}^{*}, i \leq|J|\right\}, \\
& \widetilde{\Lambda}_{k}=\left\{J \in \Lambda_{k}: \sum_{J^{\prime} \in[J] \cap \Lambda_{k}} p_{J^{\prime}}>\frac{\eta^{\alpha(k-1)}}{4 C_{1}}\right\}, \\
& \Lambda_{k}^{*}=\left\{J 1^{n} \in \mathcal{J}_{\eta^{k}}: J \in \widetilde{\Lambda}_{k}\right\}, \quad E_{k}=\bigcup\left\{S_{J}(K): J \in \Lambda_{k}^{*}\right\} .
\end{aligned}
$$

We need to show that these sets satisfy (i)-(iii). In fact $C_{\Lambda_{i}^{*}} \cap C_{\Lambda_{k}^{*}}=\emptyset$ for $1 \leq i<k$ follows from the choice of $\Lambda_{k}$. For $J 1^{n} \in \Lambda_{k}^{*}$, a similar argument to the one above shows that $n \leq q$. Hence (ii) is a direct consequence of $P\left(\Lambda_{k}\right)>1 / 2, P\left(\widetilde{\Lambda}_{k}\right)>1 / 4$ and the construction of $\Lambda_{k}^{*}$ (see the proof for the case $k=1$ ). The proof of (iii) is similar to (3.1).

In view of (ii), the process must stop at some finite step, say at $k$. Let $E=\bigcup_{i=1}^{k} E_{i}$. We have

$$
\pi\left(C_{\Lambda_{i}^{*}}\right)=\bigcup_{J \in \Lambda_{i}^{*}} S_{J}(K)=E_{i} .
$$


This implies that $\pi\left(\bigcup_{i=1}^{k} C_{\Lambda_{i}^{*}}\right)=E$ and it follows that

$$
\mu(E)=P\left(\pi^{-1} E\right) \geq P\left(\bigcup_{i=1}^{k} C_{\Lambda_{i}^{*}}\right)=\sum_{i=1}^{k} P\left(C_{\Lambda_{i}^{*}}\right) \geq \frac{1}{2} .
$$

On the other hand, (iii) implies that

$$
\mathcal{H}^{\alpha}(E) \leq \sum_{i=1}^{k} \mathcal{H}^{\alpha}\left(E_{i}\right) \leq \varepsilon \sum_{i=1}^{k} P\left(C_{\Lambda_{i}^{*}}\right)<\varepsilon .
$$

The singularity of $\mu$ is proved.

Proof of Theorem 1.2. Let $\nu=\left.\mathcal{H}^{\alpha}\right|_{K}$ and let $f$ be the Radon-Nikodym derivative of $\mu$ with respect to $\nu$. Suppose $f$ is unbounded. Then a density theorem [M, §2.14] implies that for any $M, c>0$, there exist $x \in K$ and $b>0$ such that

$$
\nu\left(\{t \in K: f(t)>M\} \cap B_{c b}(x)\right)>\nu\left(B_{c b}(x)\right) / 2 .
$$

Hence

$$
\begin{aligned}
\mu\left(B_{c b}(x)\right) & =\int_{B_{c b}(x)} f(t) d \nu(t) \geq M \nu\left(\{t \in K: f(t)>M\} \cap B_{c b}(x)\right) \\
& >M \nu\left(B_{c b}(x)\right) / 2 .
\end{aligned}
$$

Let $c=|K|$ in the above inequality. Note that $x \in K=\bigcup\left\{S(K): S \in \mathcal{A}_{b}\right\}$, and hence there exists $S \in \mathcal{A}_{b}$ such that $x \in S(K)$. That $|S(K)|=r_{S} \cdot|K| \leq$ $b c$ implies that $S(K) \subset B_{c b}(x)$. It follows that

$$
\mu\left(B_{c b}(x)\right)>M \nu(S(K)) / 2 \geq\left(r_{\min } b\right)^{\alpha} M \nu(K) / 2 .
$$

On the other hand,

$$
\begin{aligned}
\mu\left(B_{c b}(x)\right) & =\sum\left\{p_{S} \mu \circ S^{-1}\left(B_{c b}(x)\right): S \in \mathcal{A}_{b}, S(K) \cap B_{c b}(x) \neq \emptyset\right\} \\
& \leq \sum\left\{p_{S}: S \in \mathcal{A}_{b}, S(K) \cap B_{c b}(x) \neq \emptyset\right\} .
\end{aligned}
$$

By Proposition 2.1(iv), there are at most $\gamma$ terms in the last summation. We choose $M$ such that $r_{\min }^{\alpha} M \nu(K) / 2>\gamma$. Then (3.2) and the above inequality imply that there exists $S \in \mathcal{A}_{b}$ such that $p_{S}>b^{\alpha}>r_{S}^{\alpha}$. Now we consider the family of maps $\mathcal{A}_{b}$. Proposition 2.4 implies that it satisfies the WSC. The measure $\mu$ can be expressed as

$$
\mu=\sum_{S \in \mathcal{A}_{b}} p_{S} \mu \circ S^{-1}
$$

i.e., $\mu$ is a self-similar measure with respect to $\mathcal{A}_{b}$. It follows from Theorem 1.1 that $\mu$ is singular with respect to $\nu$, a contradiction.

We remark that in Theorem 1.1, the assumption that $\left\{S_{j}\right\}_{j=1}^{N}$ satisfies the WSC is essential. For the Bernoulli convolution as defined in $\S 1$ 
(with weights $p, 1-p$ instead), Peres and Solomyak [PS1] proved that if $p \in$ $[1 / 3,2 / 3]$, then $\mu$ is absolutely continuous for almost all $r \in\left[p^{p}(1-p)^{1-p}, 1\right]$. Note that $(1 / 3)^{1 / 3}(2 / 3)^{2 / 3} \approx 0.5291<2 / 3$. Hence if we take $r$ and $p$ such that $2^{2 / 3} / 3 \leq r<p=2 / 3$, we see that there are $\mu_{r}$ absolutely continuous with respect to the Lebesgue measure. Theorem 1.1 does not apply to these $\mu_{r}$.

Furthermore, it was proved in [HLW] that if $\left\{S_{j}\right\}_{j=1}^{N}$ satisfies $r_{S}<p_{S}$ for some $S$ and is absolutely continuous with respect to the Lebesgue measure (note that $\left\{S_{j}\right\}_{j=1}^{N}$ cannot satisfy the WSC in view of Theorem 1.1), then the Radon-Nikodym derivative is unbounded. In particular the above example of $\mu_{r}$ has unbounded derivatives.

It is also interesting to know that if the self-similar measure $\mu$ is absolutely continuous with respect to $\left.\mathcal{H}^{\alpha}\right|_{K}$, then the two measures must be equivalent, regardless of whether $\left\{S_{j}\right\}_{j=1}^{N}$ satisfy the WSC or not ([HLW], [MSi]).

It is well known that if the IFS satisfies the OSC then the Hausdorff dimension of $K$ satisfies $\sum_{j=1}^{N} r_{j}^{\alpha}=1$. The set of probability weights $p_{j}=r_{j}^{\alpha}$ is called the natural weight of the IFS and the corresponding self-similar measure is $\left.C \mathcal{H}^{\alpha}\right|_{K}$ with the constant $C=1 / \mathcal{H}^{\alpha}(K)$. By using Theorem 1.1, we can prove the following.

Corollary 3.2. Suppose $\left\{S_{j}\right\}_{j=1}^{N}$ satisfies the OSC and $K$ is the selfsimilar set. Then the self-similar measure $\mu$ in (1.1) is absolutely continuous with respect to $\left.\mathcal{H}^{\alpha}\right|_{K}\left(\alpha=\operatorname{dim}_{\mathrm{H}} K\right)$ if and only if the weights $p_{j}$ are $r_{j}^{\alpha}$, $j=1, \ldots, N$.

Proof. To prove the sufficiency, we observe that the OSC implies that $S_{J} \neq S_{J^{\prime}}$ for any distinct $J, J^{\prime} \in \mathcal{J}_{b}$. Hence for each $S \in \mathcal{J}_{b}, S=S_{J}$ for a unique $J$. The assumption $p_{j}=r_{j}^{\alpha}$ implies that $p_{S}=p_{J}=r_{J}^{\alpha}=r_{S}^{\alpha}$ for all $S \in \mathcal{A}_{b}, b>0$. Theorem 1.1 implies that $\mu$ is absolutely continuous with respect to $\left.\mathcal{H}^{\alpha}\right|_{K}$.

For the necessity, if $\mu$ is absolutely continuous with respect to $\left.\mathcal{H}^{\alpha}\right|_{K}$, then Theorem 1.1 implies that $p_{j} \leq r_{j}^{\alpha}, j=1, \ldots, N$. It follows from $\sum_{j=1}^{N} p_{j}=$ $\sum_{j=1}^{N} r_{j}^{\alpha}=1$ that $p_{j}=r_{j}^{\alpha}$ for all $j=1, \ldots, N$.

We can also apply the theorem to the dilation equation in wavelet theory [DL].

Corollary 3.3. Consider the dilation equation

$$
f(x)=\sum_{j=0}^{N} c_{j} f(2 x-j), \quad x \in \mathbb{R},
$$

with $c_{j} \geq 0$ and $\sum_{j} c_{2 j}=\sum_{j} c_{2 j+1}=1$. Then (3.3) has a bounded solution. 
Proof. We reformulate the equation as follows: Let $S_{j}(x)=\frac{1}{2}(x+j), p_{j}=$ $c_{j} / 2, j=0,1, \ldots, N$. Then $\left\{p_{j}\right\}_{j=0}^{N}$ satisfies $\sum_{j} p_{2 j}=\sum_{j} p_{2 j+1}=1 / 2$. Let $\mu$ be the corresponding self-similar measure defined by $\mu=\sum_{j=0}^{N} p_{j} \mu \circ S_{j}^{-1}$. It is clear that if $\mu$ is absolutely continuous with respect to the Lebesgue measure, then the Radon-Nikodym derivative is the solution of (3.3).

It is easy to see that $\left\{S_{j}\right\}_{j=1}^{N}$ satisfies the WSC [LNR]. To prove the absolute continuity, we iterate the self-similar identity for $\mu n$ times to get

$$
\mu=\sum_{j=0}^{N^{\prime}} w_{j} \mu \circ T_{j}^{-1},
$$

where $T_{j}(x)=2^{-n}(x+j), j=0,1, \ldots, N^{\prime}$ and $N^{\prime}=\left(2^{n}-1\right) N$. It follows that $\left\{w_{j}\right\}_{j=0}^{N^{\prime}}$ satisfies the summing rule

$$
\sum_{j} w_{2^{n} j}=\sum_{j} w_{2^{n} j+1}=\ldots=\sum_{j} w_{2^{n} j+2^{n}-1}=2^{-n}
$$

$\left(w_{j}=0\right.$ for $\left.j \neq 0,1, \ldots, N^{\prime}\right)$, which implies that $w_{j} \leq 2^{-n}$ for all $j=$ $0,1, \ldots, N^{\prime}$. Theorem 1.1 implies that the above $\mu$ is absolutely continuous with respect to the Lebesgue measure, and Theorem 1.2 implies that the derivative $f$ must be bounded.

It is interesting to see whether the $f$ in the above corollary will be continuous. The reader can refer to [Wa] for more detail. We further remark that Corollary 3.3 still holds if we replace the scale "2" by any $2 \leq q \in \mathbb{N}$ and modify the summing rule in an obvious way.

4. Examples. In this section, we will use some examples to demonstrate the two theorems in the last section. We also give a new class of IFS with more arbitrary contraction ratios and which satisfy the WSC.

EXAMPLE 4.1. Let $r=(\sqrt{5}-1) / 2$ be the golden ratio and let $S_{1}(x)=$ $r x, S_{2}(x)=r x+1-r$. Then the IFS satisfies the WSC [LN1]. For $0<p<1$, let $\mu_{r, p}$ be the self-similar measure defined by the weights $p$ and $1-p$. Then $\mu_{r, p}$ is singular with respect to the Lebesgue measure.

Proof. We consider three cases:

(i) $p<1-r$ or $p>r$. Then Theorem 1.1 implies that $\mu_{r, p}$ is singular.

(ii) $1-r<p<r$. Note that $S_{122}(x)=S_{211}(x)=r^{3} x+r^{2}$, and the corresponding weight satisfies $p(1-p)^{2}+p^{2}(1-p)=p(1-p)>r^{3}$. Again Theorem 1.1 implies that $\mu_{r, p}$ is singular.

(iii) $p=1-r$. Note that $S_{21211}(x)=S_{21122}(x)=S_{12222}(x)=r^{5} x+r^{4}+$ $r^{2} \in \mathcal{A}_{r^{5}}$ with weight $p^{3}(1-p)^{2}+p^{2}(1-p)^{3}+p(1-p)^{4}=2 r^{6}>r^{5}$; by symmetry, the same is true for $p=r$. Hence $\mu_{p, r}$ is singular for $p=r, 1-r$. 
For $p=1 / 2, \mu_{r, 1 / 2}$ is the well known singular case of a Bernoulli convolution [E]. The example is also true for $r^{-1}$ being a Pisot number; it is well known for $p=1 / 2$, and for $0<p<1$ it has been proved in [LNR] using a Fourier transform argument.

EXAMPle 4.2. For $0<r<1$, let

$$
S_{1}(x)=r x, \quad S_{2}(x)=r x+r, \quad S_{3}(x)=r x+1 .
$$

This family satisfies the WSC [LNR] and $\alpha=\operatorname{dim}_{\mathrm{H}} K=-\log \left(\frac{3+\sqrt{5}}{2}\right) / \log r$ [NWa]. It is interesting to observe that for any weights $\left\{p_{j}\right\}_{j=1}^{3}$, the selfsimilar measure is singular with respect to $\left.\mathcal{H}^{\alpha}\right|_{K}$.

Proof. We consider three cases:

(i) If $p_{1}>r^{\alpha}=(3-\sqrt{5}) / 2$, then Theorem 1.1 implies that $\mu$ is singular with respect to $\left.\mathcal{H}^{\alpha}\right|_{K}$.

(ii) If $\sqrt{5}-2 \leq p_{1} \leq(3-\sqrt{5}) / 2$, we notice that $S_{21}=S_{13}:=S \in \mathcal{A}_{b}$, where $b=r^{2}$, and $p_{S}=p_{2} p_{1}+p_{1} p_{3}=p_{1}\left(1-p_{1}\right)>b^{\alpha}$. The same reasoning as above shows that $\mu$ is singular.

(iii) If $p_{1}<\sqrt{5}-2$, then $p_{2}+p_{3}=1-p_{1}>3-\sqrt{5}=2 r^{\alpha}$. It follows that either $p_{2}>r^{\alpha}$ or $p_{3}>r^{\alpha}$. Once again Theorem 1.1 implies that $\mu$ is singular.

EXAMPLE 4.3. Let $S_{1}(x)=r_{1} x, S_{2}(x)=r_{2} x+1-r_{2}$, with $0<r_{1}, r_{2}<1$ and $r_{1}+r_{2}<1$. It is clear that $\left\{S_{1}, S_{2}\right\}$ satisfies the OSC. Consider the family $\left\{S_{j}\right\}_{j=1}^{3}$, where $S_{3}=S_{12}$. It is obvious that the two IFS have the same invariant set $K$; Proposition 2.4 implies that $\left\{S_{j}\right\}_{j=1}^{3}$ satisfies the WSC.

We claim that the self-similar measure $\mu$ associated with the $\left\{S_{j}\right\}_{j=1}^{3}$ is absolutely continuous with respect to $\left.\mathcal{H}^{\alpha}\right|_{K}\left(\alpha=\operatorname{dim}_{\mathrm{H}} K\right)$ if and only if $p_{1}=r_{1}^{\alpha}, p_{2}=r_{2}^{\alpha}$ and $p_{3}=0$.

Proof. The sufficiency is clear. To prove the necessity, we conclude from Theorem 1.1 that $p_{1} \leq r_{1}^{\alpha}$ and $p_{2} \leq r_{2}^{\alpha}$. By applying the self-similar identity $\mu=\sum_{j=1}^{3} p_{j} \mu \circ S_{j}^{-1}$ to $S_{1}(K)$, we get

$$
\mu=p_{1}\left(\sum_{j=1}^{3} p_{j} \mu \circ S_{j}^{-1} \circ S_{1}^{-1}\right)+p_{2} \mu \circ S_{2}^{-1}+p_{3} \mu \circ S_{3}^{-1} .
$$

Noting that $S_{j}^{-1} \circ S_{1}^{-1}=S_{1 j}^{-1}$ and $S_{3}=S_{12}$, we have

$$
\mu=p_{1}^{2} \mu \circ S_{11}^{-1}+\left(p_{1} p_{2}+p_{3}\right) \mu \circ S_{3}^{-1}+p_{1} p_{3} \mu \circ S_{13}^{-1}+p_{2} \mu \circ S_{2}^{-1} .
$$

Proposition 2.4 implies that $\left\{S_{11}, S_{3}, S_{13}, S_{2}\right\}$ satisfies the WSC; the absolute continuity of $\mu$ and Theorem 1.1 hence imply that

$$
p_{1} p_{2}+p_{3} \leq\left(r_{1} r_{2}\right)^{\alpha} .
$$


On the other hand, we recall that $S_{1}, S_{2}$ satisfies the OSC and $r_{1}^{\alpha}+r_{2}^{\alpha}=1$. Hence

$$
\begin{aligned}
p_{1} p_{2}+p_{3} & =p_{1} p_{2}+1-p_{1}-p_{2}=\left(1-p_{1}\right)\left(1-p_{2}\right) \\
& \geq\left(1-r_{1}^{\alpha}\right)\left(1-r_{2}^{\alpha}\right)=\left(r_{1} r_{2}\right)^{\alpha} .
\end{aligned}
$$

This implies that $p_{1}=r_{1}^{\alpha}, p_{2}=r_{2}^{\alpha}, p_{3}=0$.

So far all the known nontrivial examples of IFS $\left\{S_{j}\right\}_{j=1}^{N}$ with the WSC have the following property: there exists a constant $r$ such that $r_{j}=r^{k_{j}}$ for $j=1, \ldots, N$, where $k_{j} \in \mathbb{N}$ and $r_{j}$ is the contraction ratio of $S_{j}$. Example 4.3 is not of this kind as long as $\log r_{1} / \log r_{2}$ is irrational, but it is more or less a consequence of the OSC. In the rest of this section, we will give a new class of examples of IFS with quite arbitrary contraction ratios.

Proposition 4.4. Let

$$
S_{1}(x)=\varrho x, \quad S_{2}(x)=r x+\varrho(1-r), \quad S_{3}(x)=r x+1-r,
$$

with $0<\varrho<1,0<r<1$ and $\varrho+2 r-r \varrho \leq 1$. Then $\left\{S_{j}\right\}_{j=1}^{3}$ satisfies the WSC.

For convenience, we will denote by $r_{j}$ the contraction ratio of $S_{j}$, i.e., $r_{1}=\varrho, r_{2}=r_{3}=r$. We will prove the proposition after a series of lemmas. First it is straightforward to check

Lemma 4.5. Let $\left\{S_{j}\right\}_{j=1}^{3}$ be as in Proposition 4.4. Then

(i) $0=S_{1}(0)<S_{2}(0)<S_{1}(1)<S_{2}(1) \leq S_{3}(0)<S_{3}(1)=1$.

(ii) $S_{13^{k}}=S_{2^{k}}$ for any $k \in \mathbb{N}$.

It follows from (ii) that the IFS will not satisfy the OSC.

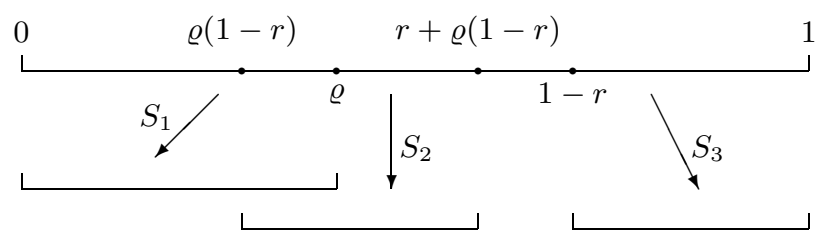

Fig. 4.1. The IFS in Proposition 4.4

Let $\Sigma^{*}=\bigcup_{n=0}^{\infty}\{1,2,3\}^{n}$. By convention we let $\{1,2,3\}^{0}:=\emptyset, j^{0}=\emptyset$, $\emptyset J=J \emptyset ; S_{\emptyset}=$ identity map, $r_{\emptyset}=1$. Let

$$
\Sigma^{\prime}=\left\{J \in \Sigma^{*}: \text { no segment of } J \text { is } 21\right\} .
$$

Then $\left\{S_{J}: J \in \Sigma^{*}\right\}=\left\{S_{J}: J \in \Sigma^{\prime}\right\}$. This follows from Lemma 4.5(ii) by replacing the segments $2^{k} 1$ in the index $J$ with $13^{k}$. Let

$$
\mathcal{J}_{b}^{\prime}=\Sigma^{\prime} \cap \mathcal{J}_{b}, \quad \mathcal{J}_{b}^{\prime \prime}=\left\{J 21 \in \mathcal{J}_{b}: J \in \Sigma^{\prime}\right\}
$$


Recall that $\mathcal{A}_{b}=\left\{S: S=S_{J}, J \in \mathcal{J}_{b}\right\}$, which means the set of distinct $S_{J}, J \in \mathcal{J}_{b}$. Let $\mathcal{A}_{b}^{\prime}$ and $\mathcal{A}_{b}^{\prime \prime}$ be defined similarly with respect to $\mathcal{J}_{b}^{\prime}$ and $\mathcal{J}_{b}^{\prime \prime}$.

LEMMA 4.6. With the above notations:

(i) if $\varrho \geq r$, then $\mathcal{A}_{b}=\mathcal{A}_{b}^{\prime}$;

(ii) if $\varrho<r$, then $\mathcal{A}_{b}=\mathcal{A}_{b}^{\prime} \cup \mathcal{A}_{b}^{\prime \prime}$.

Proof. The relation of $\mathcal{A}_{b}$ and $\mathcal{A}_{b}^{\prime}$ is obtained from replacing segments of 21 in the index with 13.

Let $J=\bar{J} 2^{k} 1$ where the last entry of $\bar{J}$ is not 2 . We replace all the 21 in $J$ by 13 and obtain $J^{\prime}=\widetilde{J} 13^{k}, \widetilde{J} \in \Sigma^{\prime}$. By Lemma 4.5(ii), $S_{J}=S_{J^{\prime}}$, and hence $r_{J}=r_{J^{\prime}}$. In case (i), $J \in \mathcal{J}_{b}$ implies $J^{\prime} \in \mathcal{J}_{b}^{\prime}$ because for $\varrho \geq r$ (i.e., $\left.r_{1}>r_{2}\right)$,

$$
r_{J} \leq b<r_{\bar{J}^{k}} \Rightarrow r_{J^{\prime}} \leq b<r_{\widetilde{J} 13^{k-1}} .
$$

In case (ii), we note that $r_{\widetilde{J} 13^{k-1}} \leq b$ may happen so that $J^{\prime} \notin \mathcal{J}_{b}^{\prime}$. In this case we keep the last pair of 21 unchanged and only replace the preceding 21 with 13 . This is the set $\mathcal{J}_{b}^{\prime \prime}$.

We consider the lexicographic order $\prec$ on $\Sigma^{*}$; for the subsets of $\Sigma^{*}$, we will use the induced order. For a subset $A \subset \Sigma^{*}$ and $J \in A$ we say that $J^{*} \in A$ is (right) adjacent to $J$ in $A$ if $J \prec J^{*}$ and there is no $\widetilde{J} \in A$ such that $J \prec \widetilde{J} \prec J^{*}$. In the following we will identify the adjacent elements in $\mathcal{J}_{b}^{\prime}$ and $\mathcal{J}_{b}^{\prime \prime}$ separately. Let $x_{J}=S_{J}(0)$ and $y_{J}=S_{J}(1)$.

Lemma 4.7. Assume that $J^{*}$ is adjacent to $J$ in $\mathcal{J}_{b}^{\prime}$.

(i) If $J=J^{\prime} 1$, then $J^{*}=J^{\prime} 2^{l}$ for some $l \geq 1$, and $x_{J}<x_{J^{*}}<y_{J}<y_{J^{*}}$.

(ii) If $J=J^{\prime} 2$, then $J^{*}=J^{\prime} 3$ and $x_{J}<y_{J} \leq x_{J^{*}}<y_{J^{*}}$.

(iii) If $J=J^{\prime} 13^{k}, k \geq 1$, then $J^{*}=J^{\prime} 2^{l}$ for some $l \geq k$, and $x_{J} \leq x_{J^{*}}<$ $y_{J}<y_{J^{*}}$.

(iv) If $J=J^{\prime} 23^{k}, k \geq 1$, then $J^{*}=J^{\prime} 31^{l}$ for some $l \geq 1$, and $x_{J}<y_{J} \leq$ $x_{J^{*}}<y_{J^{*}}$.

Proof. We only prove (i); the other cases can be proved similarly. First we note that $J^{\prime} 1 \bar{J} \notin \mathcal{J}_{b}^{\prime}$ for any $\bar{J} \neq \emptyset$, i.e., we cannot add any more indices to $J^{\prime} 1$ to be in $\mathcal{J}_{b}^{\prime}$. That $J \in \mathcal{J}_{b}^{\prime}$ implies that

$$
r_{J^{\prime}} r_{1} \leq b<r_{J^{\prime}}
$$

By the lexicographic order in $\mathcal{J}_{b}^{\prime}$, and since the adjacent element $J^{*} \in \mathcal{J}_{b}^{\prime}$ cannot contain segment 21 , the only choice is that $J^{*}=J^{\prime} 2^{l}$ with

$$
r_{J^{\prime}} r_{2}^{l} \leq b<r_{J^{\prime}} r_{2}^{l-1} \text {. }
$$


For the last part of the statement, we make use of Lemma 4.5 to get

$$
\begin{aligned}
x_{J} & =S_{J^{\prime} 1}(0)=S_{J^{\prime}}(0)<S_{J^{\prime} 2^{l}}(0)=x_{J^{*}}, \\
x_{J^{*}} & =S_{J^{\prime} 2^{l}}(0)=S_{J^{\prime} 2^{l} 1}(0)=S_{J^{\prime} 13^{l}}(0)<S_{J^{\prime} 13^{l}}(1)=S_{J^{\prime} 1}(1)=y_{J}, \\
y_{J} & =S_{J^{\prime} 13^{l}}(1)=S_{J^{\prime} 2^{l} 1}(1)<S_{J^{\prime} 2^{l}}(1)=y_{J^{*}} \text {. }
\end{aligned}
$$

Similarly, for the set $\mathcal{J}_{b}^{\prime \prime}$, we have

Lemma 4.8. Assume that $\varrho<r$ and $J^{*}$ is adjacent to $J$ in $\mathcal{J}_{b}^{\prime \prime}$.

(i) If $J=J^{\prime} 121$, then either (a) $J^{*}=J^{\prime} 12^{2} 1$ or (b) $J^{*}=J^{\prime} 2^{2} 1$.

(ii) If $J=J^{\prime} 221$, then either (a) $J^{*}=J^{\prime} 2^{3} 1$ or (b) $J^{*}=J^{\prime} 321$.

(iii) If $J=J^{\prime} 13^{k} 21, k \geq 1$, then either (a) $J^{*}=J^{\prime} 13^{k} 2^{2} 1$ or (b) $J^{*}=$ $J^{\prime} 2^{k+1} 1$.

(iv) If $J=J^{\prime} 23^{k} 21, k \geq 1$, then either (a) $J^{*}=J^{\prime} 23^{k} 2^{2} 1$ or (b) $J^{*}=$ $J^{\prime} 31^{l} 21$ for some $l \geq 0$.

Furthermore, in case (a), we have $|J|<\left|J^{*}\right|$ and $x_{J}<x_{J^{*}}<y_{J}=y_{J^{*}}$; in case (b), we have $x_{J}<y_{J}<x_{J^{*}}<y_{J^{*}}$.

Proof. We only prove (i). It is straightforward to check that if $J=$ $J^{\prime} 121 \in \mathcal{J}_{b}^{\prime \prime}$, then $J^{\prime} 1 \bar{J} 21 \notin \mathcal{J}_{b}^{\prime \prime}$, where $\bar{J} \in \Sigma^{\prime}$ contains coordinate 1 . For $\varrho<r$ (i.e., $r_{1}<r_{2}$ ), the assumption $J=J^{\prime} 121 \in \mathcal{J}_{b}^{\prime \prime}$ implies that $r_{J^{\prime} 1} r_{2} r_{1} \leq$ $b<r_{J^{\prime} 1} r_{2}$. If

$$
r_{J^{\prime} 1} r_{2}^{2} r_{1} \leq b<r_{J^{\prime} 1} r_{2}^{2}
$$

then the adjacent index is $J^{*}=J^{\prime} 12^{2} 1$; otherwise,

$$
r_{J^{\prime}} r_{2}^{2} r_{1} \leq b<r_{J^{\prime}} r_{2}^{2}
$$

and $J^{*}=J^{\prime} 2^{2} 1$. The proof for the rest of the statement is similar to the corresponding part of Lemma 4.7 .

Proof of Proposition 4.4. Let $D=[0,1]$. Then $\bigcup_{j=1}^{3} S_{j}(D) \subset D$. We consider two cases:

(i) If $\varrho \geq r$, then by Lemma 4.6, $\mathcal{A}_{b}=\mathcal{A}_{b}^{\prime}=\left\{S_{J}: J \in \mathcal{J}_{b}^{\prime}\right\}$. We rearrange $\mathcal{J}_{b}^{\prime}=\left\{J_{i}: i=1,2, \ldots, n_{b}\right\}$ so that $J_{i} \prec J_{i+1}$ and claim that

$$
S_{J_{i}}(D) \cap S_{J_{i+k}}(D)=\emptyset \quad \text { for all } i \text { and } k \geq 3 \text {. }
$$

To see this, we consider $J_{i}=J 1, J 2, J 13^{k}, J 23^{k}, k \geq 1$ as in Lemma 4.7. For each case, we can use the last assertion of Lemma 4.7 to check that $y_{J_{i}}<x_{J_{i+3}}$ and the claim follows. The claim also implies that $S_{J_{i}}(D) \cap$ $S_{J_{i-k}}(D)=\emptyset$ for all $i$ and $k \geq 3$. Hence we conclude that

$$
\#\left\{S \in \mathcal{A}_{b}: x \in S(D)\right\} \leq 5 \quad \text { for any } x \in \mathbb{R}^{d},
$$

i.e., $\left\{S_{j}\right\}_{j=1}^{3}$ satisfies the WSC with $D=[0,1]$ and $\gamma=5$.

(ii) If $\varrho<r$, then by Lemma 4.6, $\mathcal{A}_{b}=\left\{S_{J}: J \in \mathcal{J}_{b}^{\prime} \cup \mathcal{J}_{b}^{\prime \prime}\right\}$. By what we have just proved, $\#\left\{S_{J}: x \in S_{J}(D), J \in \mathcal{J}_{b}^{\prime}\right\} \leq 5$ for each $x \in D$. 
On $\mathcal{J}_{b}^{\prime \prime}$, we let $M>0$ be such that $r_{2}^{M}<r_{1}$ and we claim that

$$
S_{J_{i}}(D) \cap S_{J_{i+k}}(D)=\emptyset \quad \text { for any } i \text { and for } k \geq M .
$$

If this were not true, then part (b) in the last assertion of Lemma 4.8 cannot hold. Hence only part (a) holds; we have $y_{J_{i}}=y_{J_{i+1}}=\ldots=y_{J_{i+M}}$, and the expression for $J^{*}$ implies that

$$
r_{J_{i+M}} \leq r_{J_{i}} r_{2}^{M}<b r_{1}
$$

This contradicts the fact that $J_{i+M} \in \mathcal{J}_{b}^{\prime \prime}$ and proves the claim. It follows that

$$
\#\left\{S: x \in S(D), S \in \mathcal{A}_{b}^{\prime \prime}\right\} \leq 2 M-1 \quad \text { for any } x \in \mathbb{R}^{d} .
$$

Combining this with the above statement for $J \in \mathcal{J}_{b}^{\prime}$, we see that each $x \in \mathbb{R}^{d}$ is covered by at most $\gamma=2(M+2)$ of the $S(D), S \in \mathcal{A}_{b}$. This proves that $\left\{S_{j}\right\}_{j=1}^{3}$ satisfies the WSC.

Proposition 4.9. Let $\left\{S_{j}\right\}_{j=1}^{3}$ be the IFS in Proposition 4.4. Then the Hausdorff dimension $\alpha$ of the invariant set $K$ is given by

$$
\varrho^{\alpha}+2 r^{\alpha}-(\varrho r)^{\alpha}=1 .
$$

Moreover, the self-similar measure defined by this IFS is singular with respect to $\left.\mathcal{H}^{\alpha}\right|_{K}$.

Remark. For the case $\alpha=1, K=[0,1]$; it is interesting to note that all the singular measures induced by this IFS are singular with respect to the Lebesgue measure.

Proof. The proof makes use of an idea in $[\mathrm{H}]$. Let $K$ be the invariant set of the IFS. Then $K=\bigcup_{j=1}^{3} S_{j}(K)$. By applying the set identity to $S_{1}(K)$ and $S_{2}(K)$ and noting that $S_{13}=S_{21}$, we have

$$
K=\left(\bigcup_{i, j=1}^{2} S_{i j}(K)\right) \cup S_{23}(K) \cup S_{3}(K)
$$

Inductively,

$$
K=\left(\bigcup_{|J|=n+2} S_{J}(K)\right) \cup\left(\bigcup_{i=0}^{n} \bigcup_{|J|=i} S_{J 23}(K)\right) \cup S_{3}(K),
$$

where $J \in \bigcup_{n=0}^{\infty}\{1,2\}^{n}$. Letting $n \rightarrow \infty$, we have

$$
K=K_{0} \cup\left(\bigcup_{i=0}^{\infty} \bigcup_{|J|=i} S_{J 23}(K)\right) \cup S_{3}(K),
$$

where $K_{0}=\bigcap_{n=1}^{\infty} \bigcup\left\{S_{J}(K): J \in\{1,2\}^{n}\right\}$. 
We claim that the sets in the family

$$
\mathcal{B}=\left\{S_{J 23}(K): J \in \bigcup_{n=0}^{\infty}\{1,2\}^{n}\right\} \cup\left\{S_{3}(K)\right\}
$$

are pairwise disjoint except possibly for only one point, i.e., for any $A, B \in \mathcal{B}$, $A \cap B$ contains at most one point.

To see this, first we note that $\max \left\{S_{1}(1), S_{2}(1)\right\} \leq S_{3}(0)$, and hence if $A$ or $B$ is $S_{3}(K)$, then the claim holds. Secondly, it is easy to prove by induction that

$$
S_{j_{1} \ldots j_{n} 23}(1) \leq S_{22}(1)\left(\leq S_{23}(0)\right), \quad j_{1}, \ldots, j_{n} \in\{1,2\}, n \geq 1 .
$$

It follows that the claim holds if $A$ or $B$ is $S_{23}(K)$. Now we need only prove the claim for the subfamily

$$
\mathcal{B}_{1}=\left\{S_{J 23}(K): J \in \bigcup_{n=1}^{\infty}\{1,2\}^{n}\right\} .
$$

Let $J_{1}, J_{2} \in \bigcup_{n=1}^{\infty}\{1,2\}^{n}, J_{1} \neq J_{2}$. Without loss of generality, we assume that $\left|J_{1}\right| \leq\left|J_{2}\right|$. Then there are two possible cases:

(a) $J_{2}=J_{1} J^{\prime}$ for some $\left|J^{\prime}\right| \geq 1$;

(b) $J_{1}=J^{*} i J_{1}^{\prime}$ and $J_{2}=J^{*} j J_{2}^{\prime}$ for some $J^{*}, J_{1}^{\prime}, J_{2}^{\prime} \in \bigcup_{n=0}^{\infty}\{1,2\}^{n}$ and $i \neq j$.

In case (a), by making use of (4.4), we have

$$
S_{J_{2} 23}(1)=S_{J_{1} J^{\prime} 23}(1) \leq S_{J_{1} 22}(1) \leq S_{J_{1} 23}(0) .
$$

It follows that $S_{J_{2} 23}(K) \cap S_{J_{1} 23}(K)$ contains at most one point.

In case (b), let $D=[0,1-r]$. We note that $S_{23}(K) \subset D, \bigcup_{j=1}^{2} S_{j}(D) \subset D$ and $S_{1}(D) \cap S_{2}(D)=\{\varrho(1-r)\}$. Hence

$$
\begin{aligned}
S_{J_{1} 23}(K) \cap S_{J_{2} 23}(K) & \subset S_{J_{1}}(D) \cap S_{J_{2}}(D) \subset S_{J^{*} i}(D) \cap S_{J^{*} j}(D) \\
& =\left\{S_{J^{*}}(\varrho(1-r))\right\} .
\end{aligned}
$$

This yields the claim.

Now we will prove that $\mathcal{H}^{\alpha}\left(K_{0}\right)=0$, where $\alpha=\operatorname{dim}_{\mathrm{H}} K$. We observe that $K_{0}$ is the invariant set of $S_{1}, S_{2}$. The family satisfies the OSC as $\varrho+r<$ $\varrho+2 r-r \varrho \leq 1$. Hence $\operatorname{dim}_{\mathrm{H}} K_{0}=\alpha_{1}$, where

$$
\varrho^{\alpha_{1}}+r^{\alpha_{1}}=1 \text {. }
$$

On the other hand, the disjointness of the sets in (4.3) (except $\left.K_{0}\right)$ implies

$$
\mathcal{H}^{\alpha}(K) \geq\left(r^{\alpha}+\sum_{i=0}^{\infty} \sum\left\{r_{J 23}^{\alpha}: J \in\{1,2\}^{i}\right\}\right) \mathcal{H}^{\alpha}(K)
$$


Since $0<\mathcal{H}^{\alpha}(K)<\infty$ (Proposition 2.3), we can reduce the above inequality to $1 \geq r^{\alpha}+r^{2 \alpha} \sum_{i=0}^{\infty}\left(\varrho^{\alpha}+r^{\alpha}\right)^{i}$ (note that $r_{1}=\varrho, r_{2}=r_{3}=r$ ), so that

$$
\varrho^{\alpha}+2 r^{\alpha}-(\varrho r)^{\alpha} \leq 1
$$

Hence $\varrho^{\alpha}+r^{\alpha}<1$ and comparing it with the equation for $\alpha_{1}$, we see that $\alpha_{1}<\alpha$. This implies that $\mathcal{H}^{\alpha}\left(K_{0}\right)=0$. It follows that (4.5) is an equality and so is (4.6).

The last assertion follows from a similar argument to that in Examples 4.1 and 4.2 . Let $\mu$ be the self-similar measure corresponding to the weights $\left\{p_{j}\right\}_{j=1}^{3}$. We consider three cases:

(i) If $p_{1}>\varrho^{\alpha}$, then Theorem 1.1 implies that $\mu$ is singular.

(ii) If $1-2 r^{\alpha} \leq p_{1} \leq \varrho^{\alpha}$, then we iterate the identity $\mu=\sum_{j=1}^{3} p_{j} \mu \circ S_{j}^{-1}$ to get $\mu=\sum_{i, j=1}^{3} p_{i j} \mu \circ S_{i j}^{-1}$. Let $S=S_{13}=S_{21}$. Then we have $r_{S}=r \varrho$ and $p_{S}=p_{13}+p_{21}=p_{1}\left(1-p_{1}\right)>r_{S}^{\alpha}$. Proposition 2.4 and Theorem 1.1 imply that $\mu$ is singular.

(iii) If $p_{1}<1-2 r^{\alpha}$, then $p_{2}+p_{3}=1-p_{1}>2 r^{\alpha}$. Hence $p_{2}>r^{\alpha}$ or $p_{3}>r^{\alpha}$, and $\mu$ is singular.

5. Remarks. In both of the main theorems, the results depend a priori on the Hausdorff dimension $\alpha$ of the invariant set $K$. Unlike the OSC, there is no explicit formula for $\operatorname{dim}_{H} K$ under the WSC. In [HeLR], He et al. consider IFS of the form $S_{j}(x)=A^{-1}\left(x+d_{j}\right)$, where $A$ is an integral selfsimilar matrix and is expanding (i.e., all eigenvalues have modulus $>1$ ) and $d_{j} \in \mathbb{Z}^{d}$. By using a graph-directed system, they gave an algorithm to calculate the dimension of $K$ or the dimension of the boundary of $K$ if $K^{\circ} \neq \emptyset$. There are also other approaches to the problem [DV], [NWa]. In all these cases, the maps are assumed to have the same matrices. There is no complete solution for the IFS with the WSC yet. We also remark that for the above similitudes $S_{j}(x)=A^{-1}\left(x+d_{j}\right)$, the absolute continuity of $\mu$ can be reduced to finding the eigenvalue of a "transition" matrix constructed from the weights $\left\{p_{i}\right\}_{j=1}^{N}[\mathrm{LNR}$.

An interesting question is whether the WSC can be extended to a selfconformal family $\left\{S_{j}\right\}_{j=1}^{N}$ (i.e., the derivative $S_{j}^{\prime}(x)$ is a self-similar matrix for $x$ in a suitable domain). Our definition of WSC can be applied directly to such maps. The main problem is whether there are sufficiently many nontrivial examples (other than the affine maps and those that satisfy the OSC). We have found such an example by modifying the one in Proposition 4.4:

$$
\begin{gathered}
S_{1}(x)=\varrho x, \quad S_{2}(x)=a x^{2}+r x+\varrho(1-\varrho a-r), \\
S_{3}=\varrho a x^{2}+r x+(1-\varrho a-r),
\end{gathered}
$$

where $0<\varrho, r<1, a>0,2 a+r<1$ and $\varrho+2 r-\varrho r+\left(1+\varrho+\varrho^{2}\right) a \leq 1$. The proof is similar to Proposition 4.4 but a little more complicated. We can 
also prove that Theorems 1.1 and 1.2 hold for IFS of self-conformal maps with WSC. The details will appear elsewhere.

Acknowledgments. The authors like to thank Dr. Hui Rao and Professor Sze-Man Ngai for many valuable discussions.

\section{References}

[DL] I. Daubechies and J. Lagarias, Two-scale difference equation I. Existence and global regularity of solutions, SIAM J. Math. Anal. 22 (1991) 1338-1410.

[DV] F. Dekking and P. van der Wal, The boundary of the attractor of a recurrent iterated function system, Fractals 10 (2002), 77-89.

[E] P. Erdös, On the smoothness properties of a family of symmetric Bernoulli convolutions, Amer. J. Math. 62 (1940), 180-186.

[F] K. Falconer, The Geometry of Fractal Sets, Cambridge Univ. Press, 1985.

[FLN] A. H. Fan, K. S. Lau and S. M. Ngai, Iterated function systems with overlaps, Asian J. Math. 4 (2000), 527-552.

[G] A. Garsia, Arithmetic properties of Bernoulli's convolutions, Trans. Amer. Math. Soc. 102 (1962), 409-432.

[HeLR] X. G. He, K. S. Lau and H. Rao, Self-affine sets and graph-directed systems, Constr. Approx. 19 (2003), 373-397.

[H] T. Y. Hu, Decomposition of self-similar sets, preprint.

[HL] T. Y. Hu and K. S. Lau, Multifractal structure of convolution of the Cantor measure, Adv. Appl. Math. 27 (2001), 1-16.

[HLW] T. Y. Hu, K. S. Lau and X. Y. Wang, On the absolute continuity of a class of invariant measures, Proc. Amer. Math. Soc. 130 (2002), 759-767.

[Hut] J. E. Hutchinson, Fractals and self-similarity, Indiana Univ. Math. J. 30 (1981), 713-747.

[L] K. S. Lau, Iterated function systems with overlaps and multifractal structure, in: Trends in Probability and Related Analysis (Taipei, 1998), World Sci., River Edge, NJ, 1999, 35-76.

[LN1] K. S. Lau and S. M. Ngai, Multifractal measures and a weak separation condition, Adv. Math. 141 (1999), 45-96.

[LN2] -, 一, The $L^{q}$-dimension of the Bernoulli convolution associated with the golden ratio, Studia Math. 131 (1998), 225-251.

[LN3] -, - Second-order self-similar identities and multifractal decompositions, Indiana Univ. Math. J. 49 (2000), 925-972.

[LNR] K. S. Lau, S. M. Ngai and H. Rao, Iterated function systems with overlaps and self-similar measures, J. London Math. Soc. 63 (2001), 99-116.

[LW] K. S. Lau and X. Y. Wang, Some exceptional phenomena in multifractal formalism: Part I, preprint.

[M] P. Mattila, Geometry of Sets and Measures in Euclidean Spaces, Cambridge Univ. Press, 1995.

[MSi] R. Mauldin and K. Simon, The equivalence of some Bernoulli convolutions to Lebesgue measures, Proc. Amer. Math. Soc. 126 (1998), 2733-2736.

[NWa] S. M. Ngai and Y. Wang, Hausdorff dimension of self-similar sets with overlaps, J. London Math. Soc. 63 (2001), 655-672. 
[PSS] Y. Peres, W. Schlag and B. Solomyak, Sixty years of Bernoulli convolutions, in: Fractals and Stochastics II, C. Band, S. Graf and M. Zaehle (eds.), Progr. Probab. 46, Birkhäuser, 2000, 39-65.

[PSiS] Y. Peres, K. Simon and B. Solomyak, Self-similar sets of zero Hausdorff measure and positive packing measure, Israel J. Math. 117 (2000), 353-379.

[PS1] Y. Peres and B. Solomyak, Self-similar measures and intersection of Cantor sets, ibid. 350 (1998), 4065-4087.

[PS2] - - - Absolute continuity of Bernoulli convolutions, a simple proof, Math. Res. Lett. 3 (1996), 231-239.

[PoSi] M. Pollicott and K. Simon, The Hausdorff dimension of $\lambda$-expansions with deleted digits, Trans. Amer. Math. Soc. 347 (1995), 967-983.

[S] B. Solomyak, On the random series $\sum \pm \lambda^{n}$ (an Erdös problem), Ann. of Math. 142 (1995), 611-625.

[Wa] Y. Wang, Two-scale dilation equations and the mean spectral radius, Random Comput. Dynam. 4 (1996), 49-72.

[Z] M. Zerner, Weak separation properties for self-similar sets, Proc. Amer. Math. Soc. 124 (1996), 3529-3539.

Department of Mathematics

The Chinese University of Hong Kong, H.K.

E-mail: kslau@math.cuhk.edu.hk
Current address of Xiang-Yang Wang: School of Mathematics and Computational Science Zhong-Shan University Guang-Zhou 510275, P.R. China E-mail: multifractal@sina.com

Received January 23, 2003

Revised version August 28, 2003 